Published in final edited form as:

Proc SPIE Int Soc Opt Eng. 2019 February ; 10862: . doi:10.1117/12.2510625.

\title{
Diagnostic Performance of Receptor-Specific Surgical Specimen Staining Correlate with Receptor Expression Level
}

\author{
Broderick J. House ${ }^{a}$, Jasmin M. Schaefer ${ }^{a}$, Connor W. Barth ${ }^{a}$, Scott C. Davis ${ }^{d}$, Summer L. \\ Gibbs $^{a, b, c}$ \\ aBiomedical Engineering Department, Oregon Health \& Science University, Portland, OR 97201

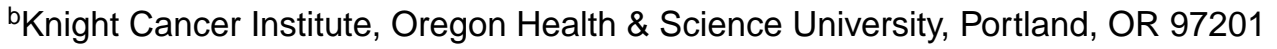 \\ 'OHSU Center for Spatial Systems Biomedicine, Oregon Health \& Science University, Portland, \\ OR 97201 \\ dThayer School of Engineering, Dartmouth College, Hanover, NH 03755
}

\begin{abstract}
Identification of tumor margins in the operating room in real time is a critical challenge for surgical procedures that serve as cancer cure. Breast conserving surgery (BCS) is particularly affected by this problem, with current re-excision rates above $25 \%$. Due to a lack of clinically available methodologies for detection of involved or close tumor margins, much effort is focused on developing intraoperative margin assessment modalities that can aid in addressing this unmet clinical need. BCS provides a unique opportunity to design contrast-based technologies that are able to assess tumor margins independent from the patient, providing a rapid pathway from bench to bedside at a much lower cost. Since resected tissue is removed from the patient's blood supply, non-specific contrast agent uptake becomes a challenge due to the lack of clearance. Therefore, a dual probe, ratiometric fluorescence imaging approach was taken in an effort to reduce nonspecific signal, and provide a modality that could demonstrate rapid, robust margin assessment on resected patient samples. Termed, dual-stain difference specimen imaging (DDSI), DDSI includes the use of spectrally unique, and fluorescently labeled target-specific, as well as non-specific biomarkers. In the present study, we have applied epidermal growth factor receptor (EGFR) targeted DDSI to tumor xenografts with variable EGFR expression levels using a previously optimized staining protocol, allowing for a quantitative assessment of the predictive power of the technique under biologically relevant conditions. Due to the presence of necrosis in the model tumors, ring analysis was employed to characterize diagnostic accuracy as measured by receiver operator characteristic (ROC) curve analysis. Our findings demonstrate the robust nature of the DDSI technique even in the presence of variable biomarker expression and spatial patterns. These results support the continued development of this technology as a robust diagnostic tool for tumor margin assessment in resected specimens during BCS.
\end{abstract}

\section{Keywords}

dual-stain difference specimen imaging; image-guided surgery; breast conserving surgery; paired agent imaging; tumor margin assessment 


\section{INTRODUCTION}

Breast cancer affects $\sim 2$ million women annually with $>626,000$ deaths in 2018 alone; making it the second leading cause of cancer mortality in women worldwide. ${ }^{1,2}$ With routine implementation of mammography screenings throughout the United States, the number of early stage breast cancers detected has increased. While early detection allows for the use of breast conserving surgery (BCS), or lumpectomy, improving cosmetic outcomes, current limits in tumor-to-normal tissue segregation impedes complete tumor resection leading to reexcision rates as high as $23 \%$ to $28 \%{ }^{3,4}$ Such rates are observed due to close or integrated tumor margins as determined through comprehensive histopathological analysis following surgery, leading to immediate follow-up surgery in order to correct the continued presence of cancer within the patient. ${ }^{5}$ Although BCS provides the cosmetic and functional benefits of tissue preservation, as compared to mastectomy, the associated follow-up surgeries lead to higher patient stress and morbidity rates, reducing the overall likelihood of a positive outcome. ${ }^{6-8}$ Therefore, total tumor resection with negative margins is essential for the improvement of BCS outcomes and the subsequent quality of life for patients.

To address this current surgical obstacle, a myriad of intraoperative margin analysis technologies have been developed, including techniques such as frozen section analysis and touch prep cytology. ${ }^{9,10}$ However, none of the current intraoperative modalities that are available have been successful in reducing the current re-excision rates, either due to limited sensitivity and/or specificity, compounded with the many challenges that come with integrating new technologies into the standard surgical workflow. ${ }^{11-16}$ As a result, an accurate and robust intraoperative tumor margin assessment technology that is able to enter the standard surgical flow for BCS is still needed.

In an effort to provide a viable modality to address the present challenges, several groups have looked into imaging of resected specimen to quantify positive margins in the operating room. One such technique developed by our lab, dual-stain difference specimen imaging (DDSI), utilizes a biomarker-specific fluorescent probe in tandem with a non-specific, spectrally distinct, untargeted isotype control probe to normalize and emphasize positive target-probe binding. ${ }^{17-24}$ Many breast cancer specific probes have been examined by numerous groups, including epidermal growth factor receptor (EGFR), human epidermal growth factor receptor 2 (HER2), estrogen receptor (ER), and CD44, in which the extracellular domain of receptors has demonstrated optimal integrity. ${ }^{17,18,23}$ The development of DDSI not only allows for tumor specific visualization with $91 \%$ sensitivity and $84 \%$ specificity in varying HER2 expressing tumors, but it has also been shown to be a viable method for easy integration into the general surgical workflow. ${ }^{17}$ With a shortened staining procedure and independence of patient contact, DDSI has promise to be integrated into the clinic in a shorter time frame than drugs that require patient contact.

Recent work within our group has demonstrated marginal change within the receiver operator characteristic (ROC) curve relationship between DDSI performance and varying EGFR-expression levels. ${ }^{25}$ Extending current work to human specimens would allow for optimization and dissemination into the general surgical environment. However, human specimens have heterogeneous biomarker expressions and varying tumor biology that cannot 
necessarily be accurately represented in preclinical mouse models. Therefore, an analysis of the robustness of the current staining methodology to such variations is needed and would allow for confirmation of the true power of DDSI for aiding in decreasing the current reexcision rates in BCS patients. In this work, DDSI was used in tumors with varying levels of necrosis and EGFR-expression in order to examine the robustness by comparing two versions of DDSI, whole tissue DDSI and cumulative ring analysis.

\section{MATERIALS AND METHODS}

\subsection{Fluorophores and Antibody Conjugates}

Alexa Fluor 647 (AF647, Thermo Fisher Scientific, Waltham, Massachusetts) and Cy3B (GE Healthcare Life Sciences, Little Chalfont, United Kingdom) were purchased in their Nhydroxysuccinimidyl ester form and solubilized in anhydrous dimethyl sulfoxide (DMSO) at $10 \mathrm{mM}$ for antibody conjugation. Cetuximab (Erbitux, Eli Lilly and Company, Branchburg, New Jersey, molecular weight $(\mathrm{MW})=152 \mathrm{kDa}$ ) was used as the targeted probe. Donkey anti-Rabbit IgG (DkRb, Jackson ImmunoResearch, West Grove, Pennsylvania, MW = 150 $\mathrm{kDa}$ ) was used as the untargeted probe. Cetuximab was conjugated to AF647, and DkRb was conjugated to $\mathrm{Cy} 3 \mathrm{~B}$. Antibody conjugations to their respective fluorophore were completed as previously described. ${ }^{17,25}$ The fluorophore-to-protein ratio of each conjugate was quantified using absorbance spectroscopy (SpectraMax M5, Molecular Devices, San Jose, California) with the antibody absorbance measured at $280 \mathrm{~nm}$ (cetuximab and DkRb extinction coefficient $\left.(\varepsilon)=210,000 \mathrm{M}^{-1} \mathrm{~cm}^{-1}\right)$, Cy3B absorbance measured at $560 \mathrm{~nm}$ (Cy3B $\left.\varepsilon=130,000 \mathrm{M}^{-1} \mathrm{~cm}^{-1}\right)$, and AF647 absorbance measured at $650 \mathrm{~nm}(\mathrm{AF} 647 \varepsilon=$ $\left.270,000 \mathrm{M}^{-1} \mathrm{~cm}^{-1}\right)$. All fluorophore-to-antibody ratios were between 3:1 and 4:1 for DDSI staining studies. The DDSI staining solution was made from a mixture of the cetuximabAF647 and DkRb-Cy3B conjugates in $1 \times$ PBS, pH 7.4, $0.1 \%$ Tween 20 , and $1 \%$ bovine serum albumin (BSA) at a final concentration $200 \mathrm{nM}$ of each antibody as measured by protein concentration.

\subsection{Cell Lines and Xenografts}

The human epidermoid carcinoma cell line A431, human pancreatic adenocarcinoma cell line AsPC-1, and human breast adenocarcinoma cell line MDA-MB-231 were cultured in Dulbeco's Modified Eagle's Medium (DMEM) (Thermo Fisher Scientific), supplemented with $1 \%$ penicillin-streptomycin-glutamine (Thermo Fisher Scientific) and 10\% fetal bovine serum (FBS, Seradigm, Sanborn, New York). All cell lines were grown to $\sim 80 \%$ confluence prior to trypsinization and preparation for suspension. The Institutional Animal Care and Use Committee at Oregon Health and Science University (OHSU) approved all animal studies. Female athymic nude mice (32 to 38 days old, homozygous 490, Charles River Laboratories, Wilmington, Massachusetts) weighing 19-21 g were used to grow xenografts of each of the three selected cell lines in the lower abdominal mammary pads. Mice were anesthetized using $100 \mathrm{mgkg}$ ketamine (Hospira Inc., Lake Forest, Illiois) and 10 mgkg xylazine (AnaSed, Shenandoah, Iowa) administered by intraperitoneal injection. Depth of anesthesia was assessed using the toe pinch method prior to tumor implantation. 
The peritoneal area was sterilized using povidone-iodine (Purdue Products, Stamford, Connecticut). A small incision ( 3 to $5 \mathrm{~mm}$ ) was made along the lateral side of each inferior nipple. Using forceps, mammary adipose was accessed through the incision and $200 \mu \mathrm{L}$ of cell suspension $\left(1 \times 10^{6}\right.$ cells $)$ were injected. The mammary adipose was inserted back through the incision, and sealed with VetbondTM (3M, St. Paul, Minnesota). Mice were monitored daily following the procedure to ensure healing for 5-7 days and then weekly for tumor growth, and overall health. Tumors were allowed to grow to a maximum size of 1.5 $\mathrm{cm}^{3}$. Each cell line was implanted into a cohort of five mice, where two tumors per mouse were implanted. This resulted in $\mathrm{n}=20$ tumor samples per cell line following bisection of the resected tumors prior to DDSI staining. ${ }^{25}$

\subsection{DDSI Staining, Imaging, and Image Processing}

Each tumor, adipose, and muscle cohort was stained and washed together following the previously published DDSI staining procedure, ${ }^{17}$ described in brief as follows. Tumor and normal tissues were blocked by incubating in $1 \mathrm{~mL}$ of $2 \%$ BSA in $1 \times$ PBS for $10 \mathrm{~min}$, followed by a 1-min incubation in $1 \mathrm{~mL}$ of $200 \mathrm{nM}$ cetuximab-AF647 and DkRb-Cy3B. The stained tissue samples were then washed in $50 \mathrm{~mL}$ of $0.1 \%$ Tween-20 in $1 \times$ PBS under gentle agitation for $5 \mathrm{~min}$. The stained tumor and normal tissues were imaged on glass slides with the bisected side facing the light source and camera. ${ }^{26}$ The resulting fluorescence images were used to calculate DDSI using custom written MatLab code (MathWorks, Natick, Massachusetts) described briefly as follows. Regions of interest (ROIs) were selected for background signal in which median signal was subtracted from the entire image. An ROI was also selected from the DDSI staining solution for intensity normalization between fluorescence channels, where each pixel value in each fluorescence image was divided by the average intensity value of the ROI selected within the DDSI staining solution. Tumor or normal tissue ROIs were selected using white light images and used to mask each fluorescence image. DDSI was calculated as $I_{\text {DDSI }}=\left(I_{\text {Targeted }}-I_{\text {Untargeted }}\right) / I_{\text {Untargeted }}$, where $I$ is the fluorescence signal intensity.

As previously described, statistical analysis was performed using MatLab. ${ }^{17}$ In brief, tumorto-normal tissue diagnostic detection was assessed through the calculation of ROC curves from DDSI images. ROC curves and corresponding area under the curve (AUC) measurement were calculated using the perfcurve function in MatLab on a pixel-by-pixel basis with individual pixel values for each tissue type used as the response variable input. Two methods were employed to quantify the DDSI ROC curves including (1) standard cumulative tissue analysis and (2) cumulative ring analysis. For the (1) standard cumulative tissue analysis the entire tumor tissue intensities was averaged in the targeted and untargeted channels, permitting DDSI ROC curve analysis. For the (2) cumulative ring analysis, an iterative analysis was again completed, where the depth of edge increased by $0.1 \mathrm{~mm}$ each iteration up to $3 \mathrm{~mm}$ from the tumor edge, targeted and untargeted intensities of each ring, which increased in thickness by $0.1 \mathrm{~mm}$ per iteration were used in the DDSI curve analysis. 25 


\subsection{IHC Staining and Microscopy}

Immediately after completion of the DDSI stain protocol, each tumor and normal tissue pair was flash frozen in optimal cutting temperature (Fisher HealthCare, Houston, Texas) compound for tissue preservation and to maintain tumor orientation. All tissue blocks were sent to the OHSU Histology Shared Resource Core where they were thawed and reembedded in paraffin, maintaining tissue orientation. The formalin-fixed paraffin embedded tissue blocks were faced prior to collection of a representative section of the tumor, adipose, and muscle pieces. IHC staining was performed on $4-\mu \mathrm{m}$ sections using an EGFR antibody (1:300, clone EP38Y, AbCam, Cambridge, Massachusetts) targeted to a different epitope to prevent steric hindrance with pre-existing labeling from cetuximab. Serial sections were used for gold standard H\&E staining. The IHC- and H\&E-stained sections provided representative EGFR expression and necrotic region tissue maps, respectively, for each tumor. Bright field images of all IHC- and H\&E-stained slides were collected at 10x magnification using the Zeiss AxioScan.Z1 (Carl Zeiss Microscopy GmbH, Jena, Germany). 25

\section{RESULTS \& DISCUSSION}

\subsection{DDSI Diagnostic Potential is Robust to Biomarker Heterogeneity}

This study examined the effects of varying EGFR-expression and necrotic regions devoid of biomarker on the diagnostic potential of DDSI. Three cell lines (A431, AsPC-1, MDAMB-231) were used to assess the diagnostic robustness of the previously reported DDSI method $^{17,25}$ within tumors containing variability in both overall EGFR-expression and heterogeneous tumor EGFR-expression patterns. With highly homogenous EGFRexpression, and little to no necrotic regions, A431 tumors provide an idealized model for comparison against the increasingly heterogeneous and necrotic tumor anatomy found within AsPC-1 and MDA-MB-231 tumor types. Following the standard DDSI staining procedure, ${ }^{17}$ fluorescence images were acquired of both targeted and untargeted probe distribution, and DDSI images were generated (Fig. 1). Utilizing an EGFR targeting antibody-fluorophore conjugate as the targeted probe, and a non-specific antibodyfluorophore conjugate as the untargeted probe, positive differentiation of tumor-to-adipose and tumor-to-muscle tissue was achieved. Importantly, DDSI images were accurate representations of EGFR expression patterns as supported through the comparison of the resulting DDSI maps to the histological gold standard IHC images (Fig. 1).

Further analyses of EGFR expression patterns reveal necrosis in AsPC-1, with centrally localized necrotic regions, and MDA-MB-231, with both central necrotic regions as well as heterogeneous necrotic spread throughout the entirety of the tumor.

To assess the impact of necrotic regions on DDSI performance, cumulative ring analysis was developed to reduce the assessment of necrotic tissue regions especially in the AsPC-1 centralized necrotic region tumors. Unlike total tissue DDSI, cumulative ring analysis utilized concentric circles starting from tumor edge and moving inward $0.1 \mathrm{~mm}$ in width until $3 \mathrm{~mm}$ was reached. As a result, when cumulative ring analysis was performed on all tumor types, the inclusion of necrosis was little to none in the AsPC-1 and A431 tumors, 
respectively. However, since the MDA-MB-231 tumors had heterogeneous necrosis, it was included in both the total tissue analysis and the cumulative ring analysis.

In order to directly compare the impact of necrotic regions on the integrity of DDSI, ROC calculated AUC values were collected and compared between the two DDSI methods. Across a range of $3 \mathrm{~mm}$, from tumor edge to center, cumulative ring analysis was utilized for all tumor types and compared to negative control muscle (Fig. 2A) or adipose tissue (Fig. 2B). The resulting AUC values were compared to see if variation was present due to differing levels of necrosis. All tumor-to-muscle AUC values were shown to be stable and close to 1.0 (Fig 2A). Additionally, results for the whole tissue and cumulative ring analysis methods for tumor-to-muscle DDSI contrast showed similar AUC values for all tumor types, demonstrating that necrosis did not impact the sensitivity nor the specificity of DDSI tumorto-muscle tissue contrast. Similarly, tumor-to-adipose AUC values across all ring analysis depths demonstrated similar AUC values to the total tissue DDSI analysis method (Fig 2B). Although the tumor-toadipose AUC values were less clustered, with A431 and AsPC-1 cell lines having AUC values at approximately 0.9 and MDA-MB-231 having an AUC value of approximately 0.7 , the robustness of the DDSI method to biomarker spatial variation was still supported. In fact, tumor-to-adipose AUC values reflect tumor expression levels accurately for the three cell lines, with low EGFR expression levels in the MDA-MB-231 tumors as compared to the A431 and AsPC-1 tumors inherently causing low tumor-toadipose contrast levels. The observed stability and equivalency seen between the ROC calculated AUC values for both total tissue and cumulative ring analysis, regardless of EGFR-expression or necrosis, supports DDSI as a robust and promising method that can provide accurate tumor-to-surrounding tissue separation in tissues with biomarker and tumor biology heterogeneity. Furthermore, the current DDSI diagnostic potential shows promise for reliable tumor margin assessment in clinical use, with high sensitivity and specificity for targeted biomarker over-expressing tumors across all tissue types.

\section{CONCLUSION}

The advancements of biomarker-targeted fluorescent imaging technologies, such as DDSI, have demonstrated that rapid tumor margin assessment in resected tissue is possible. Through systematic optimization, the DDSI methodology has proved robust to varying cancer expression levels and phenotypes, to provide an accurate representation of tumor biomarker expression levels and patterns. The current study has demonstrated that necrotic tissue cores in preclinical tumor models have little to no effect on the resulting DDSI diagnostic performance, thereby confirming that further preclinical studies with these cancer models will provide accurate results translatable to human specimens. By circumventing direct exposure to patients, DDSI is a viable option for expedient clinical approval and implementation in the surgical suite. Further work is also being pursued in an effort to reduce the current time between staining and image analysis to develop a more rapid and clinically feasible modality. As such, the next steps in bringing this type of technology to the bedside is to demonstrate its utility with fresh patient samples. To allow the translation of DDSI into the intraoperative space, extending this technology to patient samples with IHC validation is warranted in an effort for clinical optimization. Such an imaging technology would not only raise the current standards of treatment for procedures such as BCS, but it 
would also aid in the reduction of current patient morbidity, and an increase in overall patient care for BCS.

\section{REFERNECES}

[1]. Miller KD, Siegel RL, Lin CC, Mariotto AB, Kramer JL, Rowland JH, Stein KD, Alteri R and Jemal A, "Cancer treatment and survivorship statistics, 2016," CA. Cancer J. Clin 66(4), 271289 (2016). [PubMed: 27253694]

[2]. Bray F, Ferlay J, Soerjomataram I, Siegel RL, Torre LA and Jemal A, "Global cancer statistics 2018: GLOBOCAN estimates of incidence and mortality worldwide for 36 cancers in 185 countries,” CA. Cancer J. Clin 68(6), 394-424 (2018). [PubMed: 30207593]

[3]. McCahill LE, Single RM, Aiello Bowles EJ, Feigelson HS, James TA, Barney T, Engel JM and Onitilo AA, "Variability in Reexcision Following Breast Conservation Surgery," JAMA 307(5), 467 (2012). [PubMed: 22298678]

[4]. Morrow M, "Surgeon Recommendations and Receipt of Mastectomy for Treatment of Breast Cancer," JAMA 302(14), 1551 (2009). [PubMed: 19826024]

[5]. Freedman G, Fowble B, Hanlon A, Nicolaou N, Fein D, Hoffman J, Sigurdson E, Boraas M and Goldstein L, "Patients with early stage invasive cancer with close or positive margins treated with conservative surgery and radiation have an increased risk of breast recurrence that is delayed by adjuvant systemic therapy," Int. J. Radiat. Oncol. Biol. Phys 44(5), 1005-1015 (1999). [PubMed: 10421533]

[6]. Jacobs L, "Positive margins: the challenge continues for breast surgeons," Ann. Surg. Oncol 15(5), 1271-1272 (2008). [PubMed: 18320287]

[7]. Jeevan R, Cromwell DA, Trivella M, Lawrence G, Kearins O, Pereira J, Sheppard C, Caddy CM and van der Meulen JHP, "Reoperation rates after breast conserving surgery for breast cancer among women in England: retrospective study of hospital episode statistics," BMJ 345(jul12 2), e4505-e4505 (2012). [PubMed: 22791786]

[8]. Wilke LG, Czechura T, Wang C, Lapin B, Liederbach E, Winchester DP and Yao K, "Repeat Surgery After Breast Conservation for the Treatment of Stage 0 to II Breast Carcinoma: A Report From the National Cancer Data Base, 2004-2010," JAMA Surg. 149(12), 1296 (2014). [PubMed: 25390819]

[9]. Jorns JM, Visscher D, Sabel M, Breslin T, Healy P, Daignaut S, Myers JL and Wu AJ, "Intraoperative Frozen Section Analysis of Margins in Breast Conserving Surgery Significantly Decreases Reoperative Rates: One-Year Experience at an Ambulatory Surgical Center," Am. J. Clin. Pathol 138(5), 657-669 (2012). [PubMed: 23086766]

[10]. Valdes EK, Boolbol SK, Cohen J-M and Feldman SM, "Intra-operative touch preparation cytology; does it have a role in re-excision lumpectomy?," Ann. Surg. Oncol 14(3), 1045-1050 (2007). [PubMed: 17206481]

[11]. Esbona K, Li Z and Wilke LG, "Intraoperative Imprint Cytology and Frozen Section Pathology for Margin Assessment in Breast Conservation Surgery: A Systematic Review," Ann. Surg. Oncol 19(10), 3236-3245 (2012). [PubMed: 22847119]

[12]. Riedl O, Fitzal F, Mader N, Dubsky P, Rudas M, Mittlboeck M, Gnant M and Jakesz R, "Intraoperative frozen section analysis for breast-conserving therapy in 1016 patients with breast cancer,” Eur. J. Surg. Oncol. EJSO 35(3), 264-270 (2009). [PubMed: 18706785]

[13]. Saarela AO, Paloneva TK, Rissanen TJ and Kiviniemi HO, "Determinants of positive histologic margins and residual tumor after lumpectomy for early breast cancer: a prospective study with special reference to touch preparation cytology," J. Surg. Oncol 66(4), 248-253 (1997). [PubMed: 9425328]

[14]. Jorns JM, Daignault S, Sabel MS and Wu AJ, "Is Intraoperative Frozen Section Analysis of Reexcision Specimens of Value in Preventing Reoperation in Breast-Conserving Therapy?," Am. J. Clin. Pathol 142(5), 601-608 (2014). [PubMed: 25319974]

[15]. Cendán JC, Coco D and Copeland EM, "Accuracy of intraoperative frozen-section analysis of breast cancer lumpectomy-bed margins,” J. Am. Coll. Surg 201(2), 194-198 (2005). [PubMed: 16038815] 
[16]. Osako T, Nishimura R, Nishiyama Y, Okumura Y, Tashima R, Nakano M, Fujisue M, Toyozumi $\mathrm{Y}$ and Arima N, "Efficacy of intraoperative entire-circumferential frozen section analysis of lumpectomy margins during breast-conserving surgery for breast cancer," Int. J. Clin. Oncol 20(6), 1093-1101 (2015). [PubMed: 25855313]

[17]. Barth CW, Schaefer JM, Rossi VM, Davis SC and Gibbs SL, "Optimizing fresh specimen staining for rapid identification of tumor biomarkers during surgery," Theranostics 7(19), 47224734 (2017). [PubMed: 29187899]

[18]. Davis SC, Gibbs SL, Gunn JR and Pogue BW, “Topical dual-stain difference imaging for rapid intraoperative tumor identification in fresh specimens," Opt. Lett 38(23), 5184 (2013). [PubMed: 24281541]

[19]. Kang S, Wang Y "Winston,” Xu X, Navarro E, Tichauer KM and Liu JTC, "Microscopic investigation of" topically applied nanoparticles for molecular imaging of fresh tissue surfaces," J. Biophotonics 11(4), e201700246 (2018). [PubMed: 29227576]

[20]. Wang Y, Kang S, Doerksen JD, Glaser AK and Liu JTC, "Surgical Guidance via Multiplexed Molecular Imaging of Fresh Tissues Labeled with SERS-Coded Nanoparticles," IEEE J. Sel. Top. Quantum Electron. Publ. IEEE Lasers Electro-Opt. Soc 22(4) (2016).

[21]. Wang Y, Kang S, Khan A, Ruttner G, Leigh SY, Murray M, Abeytunge S, Peterson G, Rajadhyaksha M, Dintzis S, Javid S and Liu JTC, "Quantitative molecular phenotyping with topically applied SERS nanoparticles for intraoperative guidance of breast cancer lumpectomy," Sci. Rep 6(1) (2016).

[22]. Wang YW, Kang S, Khan A, Bao PQ and Liu JTC, "In vivo multiplexed molecular imaging of esophageal cancer via spectral endoscopy of topically applied SERS nanoparticles," Biomed. Opt. Express 6(10), 3714 (2015). [PubMed: 26504623]

[23]. Wang Y “Winston," Reder NP, Kang S, Glaser AK, Yang Q, Wall MA, Javid SH, Dintzis SM and Liu JTC, "Raman-Encoded Molecular Imaging with Topically Applied SERS Nanoparticles for Intraoperative Guidance of Lumpectomy," Cancer Res 77(16), 4506-4516 (2017). [PubMed: 28615226]

[24]. Wang Y, Yang Q, Kang S, Wall M and Liu J, "High-speed Raman-encoded molecular imaging of freshly excised tissue surfaces with topically applied SERRS nanoparticles," J. Biomed. Opt 23(04), 1 (2018).

[25]. Schaefer JM, Barth CW, Davis SC and Gibbs SL, "Diagnostic performance of receptor-specific surgical specimen staining correlates with receptor expression level,” J. Biomed. Opt 24(02), 1 (2019).

[26]. Hackman KM, Doddapaneni BS, Barth CW, Wierzbicki IH, Alani AWG and Gibbs SL, "Polymeric Micelles as Carriers for Nerve-Highlighting Fluorescent Probe Delivery," Mol. Pharm 12(12), 4386-4394 (2015). [PubMed: 26485440] 
A.
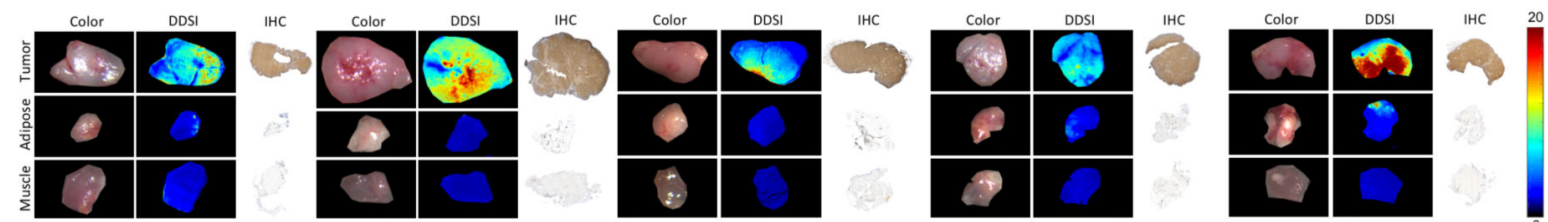

B.
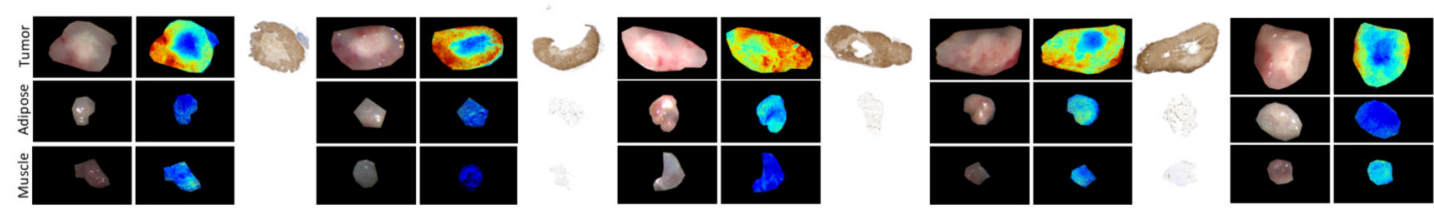

c.
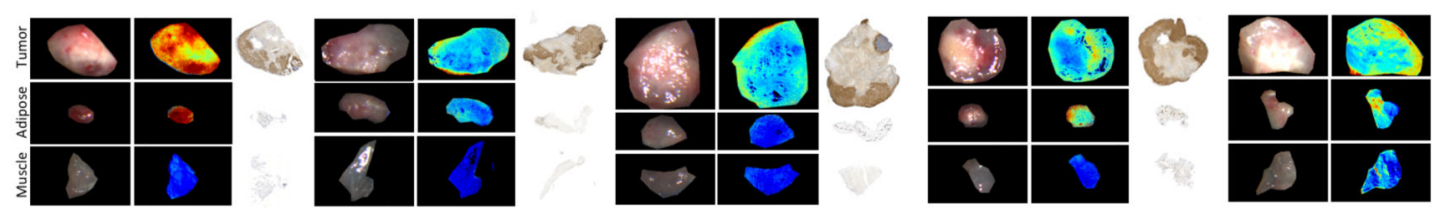

Figure 1. DDSI and IHC validation in three different EGFR-expressing xenografts.

Cohorts of each level of EGFR-expressing tumor types including (a) A431 (b) AsPC-1 (c) MDA-MB-231, with corresponding muscle and adipose tissues shown in color, DDSI, and gold-standard IHC EGFR-expression map images. All DDSI images are matched with the appropriate optimal scale at right and are representative of $n=20$. 
A.

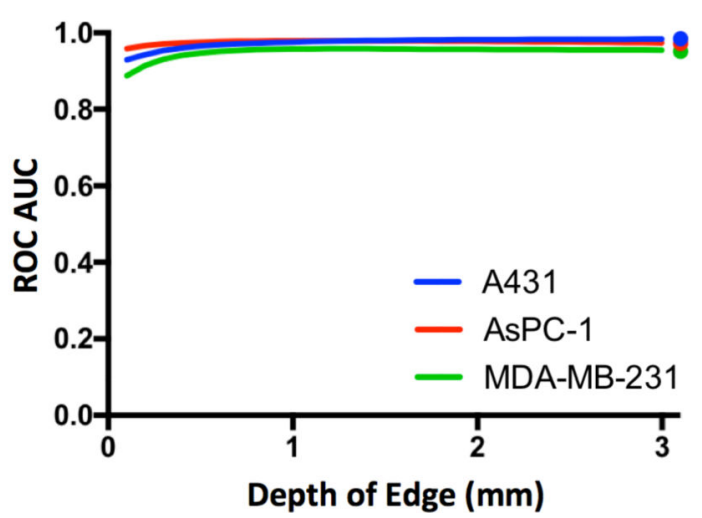

B.

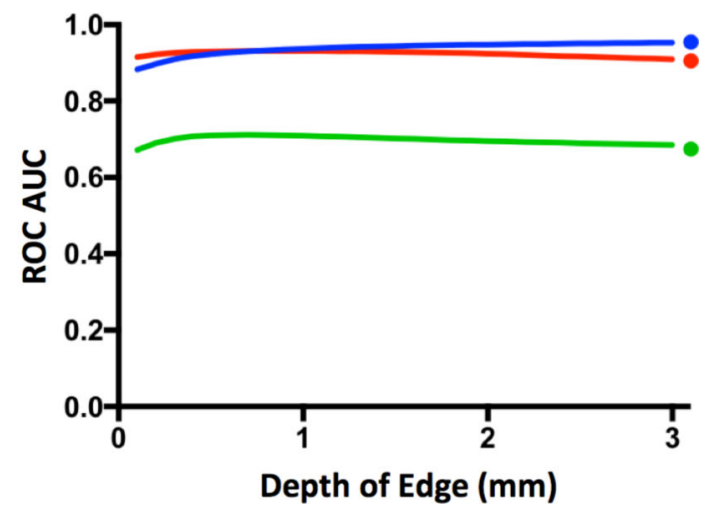

Figure 2. Cumulative whole tissue and DDSI ring analysis comparison.

Whole tissue ROC calculated AUC values for each tumor line, denoted by single points, are compared against cumulative ring analysis acquired ROC calculated AUC values for both A. tumor-to-muscle and B. tumor-to-adipose. Cumulative ring analysis was conducted on a range of 0 to $3 \mathrm{~mm}$ from tumor edge inwards by $0.1 \mathrm{~mm}$ increments. $\mathrm{ROC}=$ Receiver Operator Characteristic; AUC = Area Under the Curve. 